\title{
ALTERNATING BRANCHING PROCESSES
}

\author{
PENKA MAYSTER, ${ }^{*}$ University of Tunis
}

\begin{abstract}
We introduce the idea of controlling branching processes by means of another branching process, using the fractional thinning operator of Steutel and van Harn. This idea is then adapted to the model of alternating branching, where two Markov branching processes act alternately at random observation and treatment times. We study the extinction probability and limit theorems for reproduction by $n$ cycles, as $n \rightarrow \infty$.
\end{abstract}

Keywords: Controlled branching process; state-dependent emigration; random environment; extinction probability; limit theorem

2000 Mathematics Subject Classification: Primary 60J80

Secondary 60K05

\section{Introduction}

Our main idea is to control one branching process by means of another. We start from Sevast'yanov and Zubkov's [15] model of controlled branching processes, by defining

$$
X_{n+1}=\sum_{i=1}^{Y_{n}} \xi_{i}(n),
$$

where $Y_{n}$ is a control function depending on $X_{n}$. The traditional interpretation of the states of the Markov chain $\left(X_{n}, n=0,1,2, \ldots\right)$ is that they represent the number of particles in the $n$th generation of reproduction, defined by the independent and identically distributed (i.i.d.) random variables $\xi_{i}(n)$; see [14], [5], and [15]. The control function (which is deterministic in [15] and random in [19]) modifies the number of particles that are allowed to split in the $n$th generation. The principal assumption in Yanev's model (see [7] and [19]) is of the following form: if $X_{n}=k$ then

$$
Y_{n}=\varepsilon_{n k}[k+o(k)] \quad \text { as } k \rightarrow \infty,
$$

where $\varepsilon_{n k}$ is a family of random variables. Limiting distributions were studied in [8] and [9], convergence of controlled branching processes was investigated in [10], and multitype random control functions were introduced in [6].

Here, we propose the following autoregressive-type control:

$$
Y_{n}:=\theta \otimes X_{n}+\eta_{n}, \quad \theta \in(0,1) .
$$

The innovation sequence $\eta_{n}$ consists of i.i.d. random variables representing the immigration particles. The fractional thinning operator ' $\otimes$ ' was introduced by Steutel and van Harn [17] as 'discrete multiplication'. By definition, $\theta \otimes X_{n}=\mu\left(\tau \mid X_{n}\right)$, with $\tau=-\log \theta$, where

Received 13 August 2003; revision received 11 June 2005.

* Postal address: Institut Supérieur des Etudes Technologiques de Radès, Rue Jérusalem-Radès, B.P. 172, Radès Médina 2098, Tunisia. Email address: penka.mayster@isetr.rnu.tn 
$\mu\left(\tau \mid X_{n}\right)$ is a subcritical Markov branching process $\mu(t)$ starting with a random number of particles $\left(\mu(0)=X_{n}\right)$ and stopped at time $\tau$. We interpret $\tau$ as being the duration of a treatment time period. Actually the control process consists of testing every particle from the $n$th generation according to a dying branching process during an independent time $\tau$. We adapt this idea to the model of alternating branching. Suppose that there are two Markov branching processes, $\xi(t)$ and $\mu(t)$, acting alternately at the random observation times $\delta_{i}$ and treatment times $\tau_{i}$, respectively. We write

$$
\begin{gathered}
X_{1}=\xi_{1}\left(\delta_{1} \mid 1\right), \quad Y_{1}=\mu_{1}\left(\tau_{1} \mid X_{1}\right), \quad \ldots, \\
X_{n}=\xi_{n}\left(\delta_{n} \mid Y_{n-1}\right), \quad Y_{n}=\mu_{n}\left(\tau_{n} \mid X_{n}\right), \quad \ldots,
\end{gathered}
$$

where the random variables $\delta_{i}$ and $\tau_{i}$ are independent of the branching processes $\xi_{i}(t) \stackrel{\mathrm{D}}{=} \xi(t)$ and $\mu_{i}(t) \stackrel{\mathrm{D}}{=} \mu(t)$. Here, ' $\stackrel{\mathrm{D}}{=}$ ' denotes equality in distribution. The sequences of cycles (observation, treatment $)=\left(\delta_{i}, \tau_{i}\right)$ and (treatment, observation $)=\left(\tau_{i}, \delta_{i}\right)$ constitute a random environment for the branching mechanisms. We assume that the cycles are independent and identically distributed. Then the Markov chains $\left(X_{n}\right)$ and $\left(Y_{n}\right)$, which do not have explicit immigration, are transient. Each represents a branching process with a random environment (BPRE).

The purpose of this paper is to introduce this approach to testing the particles and to separate the branching mechanism from the random environment. Theoretically speaking we want to formulate the model in terms of BPREs.

In Section 2, we present in detail the models of autoregressive control for the GaltonWatson process and the alternating branching with immigration. Propositions 1 and 2 contain the fundamental relations. In the remaining sections, we consider only the model of alternating branching without explicit immigration. In Section 3, we describe the branching mechanism of one cycle. We note that the reproductions by one cycle of (treatment, observation) or (observation, treatment) have the same means but different variances. Therefore, the reactions $\left(X_{n}, Y_{n}\right)$, starting from the observation of one particle, and $\left(X_{n}^{\prime}, Y_{n}^{\prime}\right)$, starting from the treatment of one particle, have the same critical parameter. The problem of extinction is studied in Section 4, after a discussion of the properties of the reproduction by $n$ cycles. In the supercritical case, i.e. when the critical parameter is positive, we compare the extinction probabilities of the reactions $\left(X_{n}, Y_{n}\right)$ and $\left(X_{n}^{\prime}, Y_{n}^{\prime}\right)$. Limit theorems for the reproduction by $n$ cycles, as $n \rightarrow \infty$, are given in Section 5 (without proof) as particular cases of the well-known results of Athreya and Karlin [3].

It should be pointed out that if the observation time periods $\delta_{i}$ depend on the observed branching mechanism $\xi(t)$, then the BPRE model is no longer valid. To see this, consider the observation time $\delta$ defined by the additive functional of total progeny of the supercritical process $\xi(t)$ (see [4, p. 126]). Let

$$
y(\delta)=\int_{0}^{\delta} \xi(x) \mathrm{d} x, \quad \text { with inverse } y^{-1}(r)=\inf \{\delta: y(\delta)=r\} .
$$

This means that $\xi\left(y^{-1}(r)\right)$ is a compound Poisson process, $C(r)$. This provides a way to realize feedback control at each observation time period. Relative to the population created by the subcritical process $\mu(t)$, the influence of $C(r)$ on the successive cycles is equivalent to that of stationary immigration. The reaction $\left(X_{n}, Y_{n}\right)$ is no longer transient. The stationary solution to $\theta \otimes C(r+z)=C(r)$ has been studied by van Harn and Steutel [18] and Pakes [13]. 


\section{Models: fundamental relations}

\subsection{Autoregressive control}

A sequence $\left(X_{n}, n=0,1,2, \ldots\right)$ of $\mathbb{Z}_{+}$-valued random variables is said to be a controlled branching process with autoregressive-type control if

$$
X_{0}=0, \quad X_{n+1}=\sum_{i=1}^{Y_{n}} \xi_{i}(n), \quad Y_{n}=\theta \otimes X_{n}+\eta_{n},
$$

where $\xi_{i}(n)$ are independent copies of the integer-valued random variable $\xi$ with probabilitygenerating function (PGF) $f(s)=\mathrm{E}\left(s^{\xi}\right)$. The explicit immigration is described by the sequence of i.i.d. random variables $\eta_{i}, i=0,1,2, \ldots$, with $\eta \stackrel{\mathrm{D}}{=} \eta$ for a random variable $\eta$ whose PGF is $h(s)=\mathrm{E}\left(s^{\eta}\right)$. The thinning operator ' $\otimes$ ' is defined as follows (see [17]). Let $\theta \in(0,1)$ and let $\mu(t), t \geq 0$, be a subcritical Markov branching process starting with one particle, i.e. $\mu(0)=1$. We denote by $\mu(t \mid X)$ the same branching mechanism as $\mu(t)$, except starting with a random number of particles $X$, where $X$ is independent of $\mu(t)$. The main features of branching processes, namely the additivity by the initial condition and the independence of the evolution of particles, are manifested in the relation

$$
\mu(t \mid X)=\sum_{j=1}^{X} \mu_{j}(t), \quad \text { where } \mu_{j}(t) \stackrel{\mathrm{D}}{=} \mu(t) \text { for } j=1,2, \ldots
$$

By definition,

$$
\theta \otimes X_{n}=\mu\left(\tau \mid X_{n}\right) \quad \text { with } \tau=-\log \theta .
$$

The operator ' $\otimes$ ' becomes the analogue of scalar multiplication and incorporates the discrete nature of the variables. Relative to the population $Z_{n+1}=\sum_{i=1}^{Z_{n}} \xi_{i}(n)$ created by the reproduction law $f(s)$, the action of the thinning operator ' $\otimes$ ' is equivalent to state-dependent emigration. The PGF of $\theta \otimes X_{n}$ is given by

$$
\mathrm{E}\left(s^{\theta \otimes X_{n}}\right)=F_{n}(g(\tau, s)), \quad \tau=-\log \theta,
$$

where $F_{n}(s)=\mathrm{E}\left(s^{X_{n}}\right)$ and $g(t, s)=\mathrm{E}\left(s^{\mu(t)}\right)$. In particular, for the standard semigroup $g(t, s)=1-\mathrm{e}^{-t}(1-s)$ (see [17]), the thinning operator ' $\otimes$ ' coincides with the Bernoulli filter, i.e. $\theta \otimes X_{n}=\sum_{i=1}^{X_{n}} 1(i)$, where the $1(i) \in \operatorname{Bernoulli}(\theta)$ are i.i.d. random variables. If the branching $\xi$ occurs according to the $\operatorname{Bernoulli}(\theta)$ distribution, then the process

$$
Z_{n+1}=\sum_{i=1}^{Z_{n}} 1(i)+\eta_{n}=\theta \otimes Z_{n}+\eta_{n}
$$

which is a classical Galton-Watson process with immigration, is equivalent to the $\mathbb{Z}_{+}$-valued autoregressive process AR(1). Relevant examples and stationary distributions were considered in [1].

We now introduce the notion of a random environment using the treatment time and the observation time. We shall call $\tau=-\log \theta, \theta \in(0,1)$, the treatment time. Suppose that $\tau$ is a nonnegative random variable independent of the branching mechanisms $\xi$ and $\mu(t)$. Let $\left(\tau_{1}, \tau_{2}, \ldots\right)$ be a sequence of independent copies of $\tau$ representing the treatment time periods of the successive generations $\left(X_{1}, X_{2}, \ldots\right)$. Traditionally, the lifetime of particles in the classical 
Galton-Watson process is a constant, 1 , and reproduction is instantaneous at the end of the lifetime. We interpret the lifetime as the observation time $\delta$ and we take $\delta_{1}=\delta_{2}=\cdots=1$ for all successive generations. The sequences of pairs $\left(\tau_{i}, \delta_{i+1}\right)$ and $\left(\delta_{i}, \tau_{i}\right)$ define the cycles of reproduction for $\left(X_{n}\right)$ and $\left(Y_{n}\right)$, respectively. The transition from one cycle to another takes place as follows. Since $X_{0}=0$, the reaction is started by immigration $\eta_{0}$, such that

$$
Y_{0}=\theta \otimes X_{0}+\eta_{0}=\eta_{0}
$$

The first cycle of (treatment, observation) in the delayed sequence $d^{\prime}=\left\{\left(\tau_{i}, \delta_{i+1}\right), i=\right.$ $0,1, \ldots\}$ has the form $\left(\tau_{0}, \delta_{1}\right)=(0,1)$. We take $\tau_{0}=0$ and $X_{1}=\sum_{i=1}^{Y_{0}} \xi_{i}(0)$. Thus, the first generation observed by time $\delta_{1}=1$ is $X_{1}$, and has PGF $F_{1}(s)=h(f(s))$. The first controlled generation $Y_{1}$, allowed to split according to $\xi$, is

$$
Y_{1}=\mu_{1}\left(\tau_{1} \mid X_{1}\right)+\eta_{1}
$$

Later generations have a similar form (see (1)):

$$
X_{n+1}=\sum_{i=1}^{Y_{n}} \xi_{i}(n) \quad \text { and } \quad Y_{n}=\mu_{n}\left(\tau_{n} \mid X_{n}\right)+\eta_{n}
$$

Here, $\mu_{n}(t) \stackrel{\mathrm{D}}{=} \mu(t)$ for $n=1,2, \ldots$

Let $\sigma=\left\{\left(\delta_{i}, \tau_{i}\right)=\left(1, \tau_{i}\right), i=1,2 \ldots\right\}$ and $d^{\prime}=\left\{\left(\tau_{i}, \delta_{i+1}\right)=\left(\tau_{i}, 1\right), i=0,1,2, \ldots\right\}$. We define conditional PGFs, knowing the environments $\sigma$ and $d^{\prime}$, by

$$
F_{n}(s):=\mathrm{E}\left(s^{X_{n}} \mid d^{\prime}\right) \text { and } G_{n}(s):=\mathrm{E}\left(s^{Y_{n}} \mid \sigma\right) .
$$

By (1), we have

$$
\begin{aligned}
F_{n+1}(s) & =G_{n}(f(s))=h(f(s)) F_{n}\left(g\left(\tau_{n}, f(s)\right)\right), & & F_{0}(s)=1, \\
G_{n+1}(s) & =h(s) F_{n+1}\left(g\left(\tau_{n+1}, s\right)\right)=h(s) G_{n}\left(f\left(g\left(\tau_{n+1}, s\right)\right)\right), & & G_{0}(s)=h(s) .
\end{aligned}
$$

\subsection{Alternating branching with immigration}

Instead of the classical Galton-Watson process generated by the random variable $\xi$, we intend to control a Markov branching process $\xi(t)$ defined by the composition semigroup (and PGF) $f(t, s), t \geq 0$. Suppose that the observation time periods $\left(\delta_{1}, \delta_{2}, \ldots\right)$ form a sequence of i.i.d. copies of the nonnegative random variable $\delta$. In addition, we assume that the observation time $\delta$ and the treatment time $\tau$ are mutually independent. Let $\xi_{1}(\cdot), \xi_{2}(\cdot), \ldots$ be independent copies of $\xi(t)$ representing observed processes. As above, let $\mu_{1}(\cdot), \mu_{2}(\cdot), \ldots$, which are independent copies of $\mu(t)$, represent the treatment processes, and let $\eta_{0}, \eta_{1}, \ldots$ represent the immigration particles. We define

$$
\begin{aligned}
& X_{0}=0, \quad X_{n}=\xi_{n}\left(\delta_{n} \mid Y_{n-1}\right), \quad n=1,2, \ldots, \\
& Y_{0}=\eta_{0}, \quad Y_{n}=\mu_{n}\left(\tau_{n} \mid X_{n}\right)+\eta_{n}, \quad n=1,2, \ldots
\end{aligned}
$$

Given the environment $\sigma=\left\{\left(\delta_{i}, \tau_{i}\right), i=1,2, \ldots\right\}$, the reproduction by the $n$th cycle of (observation, treatment $)=\left(\delta_{n}, \tau_{n}\right)$ has random PGF

$$
\varphi_{n}(s, \sigma)=f\left(\delta_{n}, g\left(\tau_{n}, s\right)\right):=f \circ g \text { for } n=1,2, \ldots
$$


Indeed, a particle observed by time $\delta_{n}$ and its offspring tested by time $\tau_{n}$ are transformed into a random number, $\sum_{j=1}^{\xi\left(\delta_{n}\right)} \mu_{j}\left(\tau_{n}\right)$, of particles. Given the environment $d^{\prime}=\left\{\left(\tau_{i}, \delta_{i+1}\right), i=\right.$ $0,1, \ldots\}$, the reproduction by the $n$th delayed cycle of (treatment, observation) $=\left(\tau_{n-1}, \delta_{n}\right)$ has random PGF

$$
\psi_{n}\left(s, d^{\prime}\right)=g\left(\tau_{n-1}, f\left(\delta_{n}, s\right)\right):=g \circ f \text { for } n=2,3, \ldots,
$$

with

$$
\psi_{1}\left(s, d^{\prime}\right)=g\left(0, f\left(\delta_{1}, s\right)\right)=f\left(\delta_{1}, s\right) \quad \text { since } g(0, s)=s .
$$

Naturally, the delayed cycles are i.i.d. only for $n=2,3, \ldots$ The explicit immigration particles $\eta_{n-1}$ arriving at the time $\sum_{i=1}^{n-1}\left(\delta_{i}+\tau_{i}\right)$ reproduce during the observation time period $\delta_{n}$ according to the Markov branching process $\xi_{n}(\cdot)$ and increase the population $X_{n}$ by $\xi_{n}\left(\delta_{n} / \eta_{n-1}\right)$ particles having random PGF $h\left(f\left(\delta_{n}, s\right)\right):=h \circ f$.

In general, the random environment and branching mechanisms are defined on the same probability space $(\Omega, \Sigma, \mathrm{P})$. We denote by $\mathrm{E}$ the mean and the conditional mean. All equalities between random variables are in distribution and almost sure, if it is not mentioned. We can now state the following result.

Proposition 1. If the random environments $\sigma$ and $d^{\prime}$ consist of i.i.d. random cycles independent of the branching mechanisms $\xi(t)$ and $\mu(t)$, then the sequences $\left(X_{n}\right)$ and $\left(Y_{n}\right)$ are Markov chains representing Galton-Watson processes with immigration in a random environment. The sequence $\left(X_{n}\right)$ has random reproduction law $\psi=g \circ f$ and immigration law $h \circ f$. The sequence $\left(Y_{n}\right)$ has random reproduction law $\varphi=f \circ g$ and immigration law $h$. The fundamental relations, analogous to (2) and (3), are

$$
\begin{array}{rlrl}
\mathrm{E}\left(s^{X_{n+1}} \mid d^{\prime}\right):=F_{n+1}(s)=h\left(f\left(\delta_{n+1}, s\right)\right) F_{n}\left(\psi_{n+1}\left(s, d^{\prime}\right)\right), & & F_{0}(s)=1, \\
\mathrm{E}\left(s^{Y_{n+1}} \mid \sigma\right):=G_{n+1}(s)=h(s) G_{n}\left(\varphi_{n+1}(s, \sigma)\right), & G_{0}(s)=h(s) .
\end{array}
$$

As $\left(X_{n}\right)$ and $\left(Y_{n}\right)$ are Markov chains, we can define the following semiregenerative processes with Markov-type cycle dependence:

$$
\left(X_{n}, \tau_{n}, \mu_{n}(r) ; 0 \leq r \leq \tau_{n}\right), \quad\left(Y_{n}, \delta_{n+1}, \xi_{n+1}(z) ; 0 \leq z \leq \delta_{n+1}\right) .
$$

We consider two renewal epoch processes $\left(S_{n}\right)$ and $\left(S_{n}^{\prime}\right)$, as follows:

$$
\begin{array}{lll}
S_{0}=0, & S_{n}=S_{n-1}+\left(\delta_{n}+\tau_{n}\right), & n=1,2, \ldots, \\
S_{0}^{\prime}=0, & S_{n}^{\prime}=S_{n-1}^{\prime}+\left(\tau_{n-1}+\delta_{n}\right), & n=1,2, \ldots, \quad \tau_{0}=0 .
\end{array}
$$

Then we define the alternating regenerative process

$$
Z(x)= \begin{cases}\xi_{n+1}\left(x-S_{n} \mid Y_{n}\right) & \text { if } S_{n} \leq x<S_{n+1}^{\prime}, \\ \mu_{n+1}\left(x-S_{n+1}^{\prime} \mid X_{n+1}\right) & \text { if } S_{n+1}^{\prime} \leq x<S_{n+1}\end{cases}
$$

The initial conditions of the cycles are

$$
\xi_{n+1}\left(0+\mid Y_{n}\right)=Y_{n} \text { and } \mu_{n+1}\left(0+\mid X_{n+1}\right)=X_{n+1} .
$$

Let $A(y)=\mathrm{P}\left(\delta_{i} \leq y\right)$ and $B(y)=\mathrm{P}\left(\tau_{i} \leq y\right)$ be probability distribution functions. Consider the unconditional PGFs $\bar{G}_{n}(s)=\mathrm{E}\left(G_{n}(s)\right)$ and $\bar{F}_{n}(s)=\mathrm{E}\left(F_{n}(s)\right)$. By the total probability formula, we have the following result, where by ' $*$ ' we denote the convolution of probability distribution functions. 
Proposition 2. The unconditional PGF $H(x, s)=\mathrm{E}\left(s^{Z(x)}\right), x>0$, satisfies the renewal-type relation

$$
\begin{aligned}
H(x, s)= & h(f(x, s))[1-A(x)]+\sum_{n=1}^{\infty} \int_{0}^{x} \bar{G}_{n}(f(x-y, s))[1-A(x-y)](A * B)^{* n}(\mathrm{~d} y) \\
& +\sum_{n=0}^{\infty} \int_{0}^{x} \bar{F}_{n+1}(g(x-y, s))[1-B(x-y)](A * B)^{* n} * A(\mathrm{~d} y) .
\end{aligned}
$$

(See [11, pp. 201-215] and [12].)

\section{Reproduction by one cycle}

We intend to compare the reproductions by the cycles (observation, treatment) and (treatment, observation). Let $\sigma^{\prime}=\left\{\left(\tau_{i}, \delta_{i}\right), i=1,2, \ldots\right\}$. Consider the random PGFs

$$
\varphi_{i}(s, \sigma)=f\left(\delta_{i}, g\left(\tau_{i}, s\right)\right) \quad \text { and } \quad \psi_{i}\left(s, \sigma^{\prime}\right)=g\left(\tau_{i}, f\left(\delta_{i}, s\right)\right) .
$$

Let $u(s)$ and $v(s)$ be the infinitesimal generating functions of the composition semigroups $f(t, s)$ and $g(t, s)$, respectively (see [4, p. 106] and [14, p. 27]). We suppose that $f(t, s)$ is supercritical (i.e. $u^{\prime}(1)>0$ ) and $g(t, s)$ is subcritical (i.e. $v^{\prime}(1)<0$ ). Traditionally, $q$ denotes the extinction probability, that is, the smallest root in $[0,1]$ of the equation $u(s)=0$ or $v(s)=0$. The supercritical process $\xi(t)$ has extinction probability $q \equiv q(\xi)<1$ and the subcritical process $\mu(t)$ has extinction probability $q(\mu)=1$. The role of the random environment $\left(\delta_{n}, \tau_{n}\right)$ can be seen explicitly in the following representations of the PGFs, where a prime denotes differentiation:

$$
f(\delta, s)=\mathcal{A}^{-1}\left(\mathrm{e}^{u^{\prime}(q) \delta} \mathcal{A}(s)\right), \quad g(\tau, s)=\mathcal{B}^{-1}\left(\mathrm{e}^{v^{\prime}(1) \tau} \mathcal{B}(s)\right) .
$$

Here,

$$
\mathcal{B}(s):=\exp \left\{v^{\prime}(1) \int_{0}^{s} \frac{\mathrm{d} x}{v(x)}\right\}, \quad s \in[0,1), \quad \mathcal{B}(0)=1,
$$

and

$$
\mathcal{A}(s):=\exp \left\{u^{\prime}(q) \int_{q}^{s} \frac{\mathrm{d} x}{u(x)}\right\}, \quad s \in[0,1), \quad \mathcal{A}(q)=0 .
$$

Clearly, $\mathcal{B}$, which has inverse $\mathcal{B}^{-1}$, is decreasing (see [13]), while the function $\mathcal{A}$, which has inverse $\mathcal{A}^{-1}$, is increasing and satisfies $\mathcal{A}(1)=\infty$ and $\mathcal{A}(0)<0$ (see [4, p. 115]). The functions $\mathcal{A}(s)$ and $\mathcal{B}(s)$ contain information about the asymptotic behavior of the nonlinear semigroups $f(t, s)$ and $g(t, s)$.

Proposition 3. Let the random environments $\sigma=\left\{\left(\delta_{i}, \tau_{i}\right), i=1,2, \ldots\right\}$ and $\sigma^{\prime}=\left\{\left(\tau_{i}, \delta_{i}\right)\right.$, $i=1,2, \ldots\}$ be independent of the branching mechanisms $\xi(t)$ and $\mu(t)$. Suppose that $\delta_{i} \stackrel{\mathrm{D}}{=} \delta$ and $\tau_{i} \stackrel{\mathrm{D}}{=} \tau, i=1,2, \ldots$, are sequences of i.i.d. positive random variables with finite means. Denote by $\zeta_{n}$ and $\zeta_{n}^{\prime}$ integer-valued random variables representing reproductions by the $n$th cycles of $\sigma$ and $\sigma^{\prime}$, respectively. Then the sequences of random PGFs

$$
\varphi_{n}(s, \sigma):=\mathrm{E}\left(s^{\zeta_{n}} \mid \sigma\right) \text { and } \psi_{n}\left(s, \sigma^{\prime}\right):=\mathrm{E}\left(s^{\zeta_{n}^{\prime}} \mid \sigma^{\prime}\right)
$$

are independent copies of $\varphi=f \circ g$ and $\psi=g \circ f$, where

$$
\begin{aligned}
\varphi(s, \sigma) & =\mathcal{A}^{-1}\left(\mathrm{e}^{u^{\prime}(q) \delta} \mathcal{A} \circ \mathcal{B}^{-1}\left(\mathrm{e}^{v^{\prime}(1) \tau} \mathcal{B}(s)\right)\right), \\
\psi\left(s, \sigma^{\prime}\right) & =\mathscr{B}^{-1}\left(\mathrm{e}^{v^{\prime}(1) \tau} \mathcal{B} \circ \mathcal{A}^{-1}\left(\mathrm{e}^{u^{\prime}(q) \delta} \mathcal{A}(s)\right)\right) .
\end{aligned}
$$


The reproductions $\zeta \stackrel{\mathrm{D}}{=} \zeta_{n}$ and $\zeta^{\prime} \stackrel{\mathrm{D}}{=} \zeta_{n}^{\prime}$ by the cycles (observation, treatment) and (treatment, observation) have the same means but different variances, given the respective environments $\sigma$ and $\sigma^{\prime}$. These are as follows:

$$
\begin{gathered}
\mathrm{E}(\zeta \mid \sigma)=\mathrm{E}\left(\zeta^{\prime} \mid \sigma^{\prime}\right)=\exp \left\{u^{\prime}(1) \delta+v^{\prime}(1) \tau\right\}, \\
\operatorname{var}(\zeta \mid \sigma)=\operatorname{var}(\xi(t) \mid t=\delta) \mathrm{E}(\mu(t) \mid t=\tau)^{2}+\mathrm{E}(\xi(t) \mid t=\delta) \operatorname{var}(\mu(t) \mid t=\tau), \\
\operatorname{var}\left(\zeta^{\prime} \mid \sigma^{\prime}\right)=\operatorname{var}(\mu(t) \mid t=\tau) \mathrm{E}(\xi(t) \mid t=\delta)^{2}+\mathrm{E}(\mu(t) \mid t=\tau) \operatorname{var}(\xi(t) \mid t=\delta) .
\end{gathered}
$$

Let the observation times $\delta_{i}$ and treatment times $\tau_{i}$, being mutually independent, have probability distribution functions $A(\cdot)$ and $B(\cdot)$, respectively. Consider the unconditional PGFs of the reproduction by one cycle (of each type):

$$
\bar{\varphi}(s):=\mathrm{E}(f(\delta, g(\tau, s))), \quad \bar{\psi}(s):=\mathrm{E}(g(\tau, f(\delta, s))) .
$$

Let

$$
\bar{f}(s):=\mathrm{E}(f(\delta, s))=\int f(x, s) A(\mathrm{~d} x) \quad \text { and } \quad \bar{g}(s):=\mathrm{E}(g(\tau, s))=\int g(y, s) B(\mathrm{~d} y) .
$$

Jensen's inequality and convexity of the PGFs provide the inequalities

$$
\bar{\varphi} \geq \bar{f} \circ \bar{g} \quad \text { and } \quad \bar{\psi} \geq \bar{g} \circ \bar{f} .
$$

Proposition 4. If the observation and treatment time periods follow exponential distributions, i.e. $A(\mathrm{~d} x)=\alpha \mathrm{e}^{-\alpha x} \mathrm{~d} x$ and $B(\mathrm{~d} x)=\beta \mathrm{e}^{-\beta x} \mathrm{~d} x, \alpha>0, \beta>0$, then

$$
\bar{g}(s)=s+[\mathcal{B}(s)]^{\beta / v^{\prime}(1)} \int_{s}^{1}[\mathcal{B}(y)]^{-\beta / v^{\prime}(1)} \mathrm{d} y
$$

and

where $u(q)=0$.

$$
\bar{f}(s)=s+[\mathcal{A}(s)]^{\alpha / u^{\prime}(q)} \int_{s}^{q}[\mathcal{A}(x)]^{-\alpha / u^{\prime}(q)} \mathrm{d} x
$$

Proof. We will prove the result for $\bar{f}(s)$; the proof for $\bar{g}(s)$ is similar. By (4), we have

$$
\mathrm{e}^{u^{\prime}(q) t}=\frac{\mathcal{A}(f(t, s))}{\mathcal{A}(s)} \text { and } \mathrm{e}^{-\alpha t}=\left[\frac{\mathcal{A}(f(t, s))}{\mathcal{A}(s)}\right]^{-\alpha / u^{\prime}(q)} .
$$

By the backward equation and the change of variable $x=f(t, s)$, where $f(0, s)=s$ and $f(\infty, s)=q$, we obtain

$$
\begin{aligned}
\bar{f}(s) & =\int_{0}^{\infty} f(t, s) \alpha \mathrm{e}^{-\alpha t} \mathrm{~d} t=\int_{0}^{\infty} f(t, s) \alpha\left[\frac{\mathcal{A}(f(t, s))}{\mathcal{A}(s)}\right]^{-\alpha / u^{\prime}(q)} \frac{\partial f(t, s)}{\partial t} \frac{\mathrm{d} t}{u(f(t, s))} \\
& =\alpha[\mathcal{A}(s)]^{\alpha / u^{\prime}(q)} \int_{s}^{q} x[\mathcal{A}(x)]^{-\alpha / u^{\prime}(q)} \frac{\mathrm{d} x}{u(x)} .
\end{aligned}
$$

Moreover, $1 / u(x)=\mathcal{A}^{\prime}(x) / \mathcal{A}(x) u^{\prime}(q)$ and $\mathcal{A}(q)=0$. On integrating by parts, we obtain

$$
\begin{aligned}
\bar{f}(s) & =\frac{\alpha}{u^{\prime}(q)}[\mathcal{A}(s)]^{\alpha / u^{\prime}(q)} \int_{s}^{q} x[\mathcal{A}(x)]^{-\alpha / u^{\prime}(q)-1} \mathrm{~d}(\mathcal{A}(x)) \\
& =-[\mathcal{A}(s)]^{\alpha / u^{\prime}(q)}\left\{\left.x[\mathcal{A}(x)]^{-\alpha / u^{\prime}(q)}\right|_{x=s} ^{x=q}-\int_{s}^{q}[\mathcal{A}(x)]^{-\alpha / u^{\prime}(q)} \mathrm{d} x\right\} \\
& =s+[\mathcal{A}(s)]^{\alpha / u^{\prime}(q)} \int_{s}^{q}[\mathcal{A}(x)]^{-\alpha / u^{\prime}(q)} \mathrm{d} x
\end{aligned}
$$


Example 1. (Bernoulli filter and Yule process.) Let $\delta$ and $\tau$ follow the exponential distributions from Proposition 4. For the standard semigroup, we have

$$
g(t, s)=1-\mathrm{e}^{-t}(1-s) \quad \text { and } \quad \bar{g}(s)=\frac{1+\beta s}{1+\beta}, \quad \beta>0 .
$$

For the Yule process $u(s)=a\left(s^{2}-s\right)$, we have $u^{\prime}(1)=a>0$ (see [4, p. 109]). The extinction probability is $q=0$ and we have $a=-u^{\prime}(q)$ and

$$
f(t, s)=1-\frac{\mathrm{e}^{a t}(1-s)}{1+(1-s)\left[\mathrm{e}^{a t}-1\right]} .
$$

The functions $\mathcal{A}$ and $\mathcal{A}^{-1}$ are given by

$$
\mathcal{A}(s)=\frac{s}{1-s} \quad \text { and } \quad \mathcal{A}^{-1}(s)=\frac{s}{1+s},
$$

while the unconditional PGF is given by

$$
\bar{f}(s)=s+\left(\frac{1-s}{s}\right)^{\alpha / a} \int_{s}^{0}\left(\frac{x}{1-x}\right)^{\alpha / a} \mathrm{~d} x, \quad \alpha>0 .
$$

In particular, if $\alpha=2 a$ then

$$
\bar{f}(s)=s+\left(\frac{1-s}{s}\right)^{2}\left[1-s-\frac{1}{1-s}-2 \log (1-s)\right]=\sum_{k=1}^{\infty} \frac{4 s^{k}}{k(k+1)(k+2)} .
$$

The first derivative of this is

$$
\bar{f}^{\prime}(s)=\frac{2}{s^{2}}\left[2-s+2 \frac{1-s}{s} \log (1-s)\right]=\sum_{k=0}^{\infty} \frac{4 s^{k}}{(k+2)(k+3)},
$$

meaning that $\bar{f}^{\prime}(1)=2$ while $\bar{f}^{\prime \prime}(1)=4\left[-\frac{2}{3}+\sum_{n=4}^{\infty} 1 / n\right]$, on the other hand, is infinite.

\section{Extinction probability}

\subsection{Reproduction by $\boldsymbol{n}$ cycles}

Here, we consider the model of alternating branching without explicit immigration. Obviously, the initial conditions will play an important role. Suppose that the reaction $\left(X_{n}, Y_{n}\right)$ starts with one particle observed by time $\delta_{1}$. We have

$$
\begin{array}{rlrl}
Y_{0} & =1, & X_{1} & =\xi_{1}\left(\delta_{1} \mid 1\right), \\
Y_{n} & =\mu_{n}\left(\tau_{n} \mid X_{n}\right), & X_{n+1} & =\xi_{n+1}\left(\delta_{n+1} \mid Y_{n}\right), \\
& \ldots
\end{array}
$$

We shall compare it with the reaction $\left(X_{n}^{\prime}, Y_{n}^{\prime}\right)$, starting with one particle treated by time $\tau_{1}$. We define

$$
\begin{aligned}
& X_{0}^{\prime}=1, \quad Y_{1}^{\prime}=\mu_{1}\left(\tau_{1} \mid 1\right), \quad \ldots, \\
& X_{n}^{\prime}=\xi_{n}\left(\delta_{n} \mid Y_{n}^{\prime}\right), \quad Y_{n+1}^{\prime}=\mu_{n+1}\left(\tau_{n+1} \mid X_{n}^{\prime}\right), \quad \ldots
\end{aligned}
$$


The Markov chains $\left(Y_{n}\right)$ and $\left(X_{n}^{\prime}\right)$ are respectively conditioned on the random environment of the cycles

$$
\begin{aligned}
\sigma & =\left\{(\text { observation, treatment })=\left(\delta_{i}, \tau_{i}\right), i=1,2, \ldots\right\}, \\
\sigma^{\prime} & =\left\{(\text { treatment, observation })=\left(\tau_{i}, \delta_{i}\right), i=1,2, \ldots\right\} .
\end{aligned}
$$

Furthermore, the Markov chains $\left(X_{n}\right)$ and $\left(Y_{n}^{\prime}\right)$ are respectively conditioned on the delayed random environment of the cycles

$$
\begin{aligned}
d^{\prime} & =\left\{(\text { treatment, observation })=\left(\tau_{i}, \delta_{i+1}\right), i=0,1, \ldots, \tau_{0}=0\right\}, \\
d & =\left\{(\text { observation, treatment })=\left(\delta_{i}, \tau_{i+1}\right), i=0,1, \ldots, \delta_{0}=0\right\} .
\end{aligned}
$$

The first cycle of the delayed random environment brings reproduction with the following PGFs:

$$
\begin{aligned}
& \varphi_{1}(s, d)=f\left(\delta_{0}, g\left(\tau_{1}, s\right)\right)=f\left(0, g\left(\tau_{1}, s\right)\right)=g\left(\tau_{1}, s\right), \quad \text { since } f(0, s)=s, \\
& \psi_{1}\left(s, d^{\prime}\right)=g\left(\tau_{0}, f\left(\delta_{1}, s\right)\right)=g\left(0, f\left(\delta_{1}, s\right)\right)=f\left(\delta_{1}, s\right), \quad \text { since } g(0, s)=s \text {. }
\end{aligned}
$$

We remark that, for $n=2,3, \ldots$, the delayed cycles are equal in distribution to the 'regular' one, since $\left(\delta_{i}, \tau_{i+1}\right) \stackrel{\mathrm{D}}{=}\left(\delta_{i}, \tau_{i}\right)$ and $\left(\tau_{i}, \delta_{i+1}\right) \stackrel{\mathrm{D}}{=}\left(\tau_{i}, \delta_{i}\right)$, for $i=1,2, \ldots$

Denote by $T$ the shift operator on the random environment, i.e. translation by one cycle, defined by $T d^{\prime}=\left\{\left(\tau_{i}, \delta_{i+1}\right), i=1,2, \ldots\right\}, T d=\left\{\left(\delta_{i}, \tau_{i+1}\right), i=1,2 \ldots\right\}, T \sigma=$ $\left\{\left(\delta_{i}, \tau_{i}\right), i=2,3, \ldots\right\}$, and $T \sigma^{\prime}=\left\{\left(\tau_{i}, \delta_{i}\right), i=2,3 \ldots\right\}$. As the random environment consists of i.i.d. cycles, they have the following properties.

(a) The random environments $\sigma$ and $\sigma^{\prime}$ are stationary ergodic processes (see [2]). The reproduction by the first $n$ cycles is equal in distribution to the reproduction by any $n$ successive cycles. Analytically, this property can be expressed by the composition of the random PGFs of $n$ cycles. That is, if we let

$$
\phi_{n}(s, \sigma):=\mathrm{E}\left(s^{Y_{n}} \mid \sigma\right)=\varphi_{1} \circ \varphi_{2} \circ \cdots \circ \varphi_{n}(s, \sigma)
$$

then, for each $s, 0 \leq s \leq 1$, we have

$$
\phi_{n}(s, T \sigma):=\varphi_{2} \circ \varphi_{3} \circ \cdots \circ \varphi_{n+1}(s, \sigma) \stackrel{\mathrm{D}}{=} \phi_{n} \rightarrow(s, \sigma) .
$$

Furthermore, if we let

$$
\psi_{n}\left(s, \sigma^{\prime}\right):=\mathrm{E}\left(s^{X_{n}^{\prime}} \mid \sigma^{\prime}\right)=\psi_{1} \circ \psi_{2} \circ \cdots \circ \psi_{n}\left(s, \sigma^{\prime}\right)
$$

then, for each $s, 0 \leq s \leq 1$, we have

$$
\psi_{n}\left(s, T \sigma^{\prime}\right):=\psi_{2} \circ \psi_{3} \circ \cdots \circ \psi_{n+1}\left(s, \sigma^{\prime}\right) \stackrel{\mathrm{D}}{=} \psi_{n} \rightarrow\left(s, \sigma^{\prime}\right) .
$$

For the delayed random environments $d$ and $d^{\prime}$, we can similarly write

$$
\begin{aligned}
\mathrm{E}\left(s^{X_{n}} \mid d^{\prime}\right) & :=f\left(\delta_{1}, \psi_{n-1}\left(s, T d^{\prime}\right)\right) \stackrel{\mathrm{D}}{=} f\left(\delta_{1}, \psi_{n-1}\left(s, \sigma^{\prime}\right)\right), \\
\mathrm{E}\left(s^{Y_{n}^{\prime}} \mid d\right) & :=g\left(\tau_{1}, \phi_{n-1}(s, T d)\right) \stackrel{\mathrm{D}}{=} g\left(\tau_{1}, \phi_{n-1}(s, \sigma)\right) .
\end{aligned}
$$

(b) The random environments $\sigma$ and $\sigma^{\prime}$ are exchangeable processes. By definition, a stationary ergodic process $\sigma=\left\{\left(\delta_{i}, \tau_{i}\right), i=1,2, \ldots\right\}$ is said to be exchangeable if the random vectors

$$
\left(\left(\delta_{i}, \tau_{i}\right), \ldots,\left(\delta_{i+n}, \tau_{i+n}\right)\right) \text { and }\left(\left(\delta_{i+n}, \tau_{i+n}\right), \ldots,\left(\delta_{i}, \tau_{i}\right)\right)
$$


are identically distributed for each $i \geq 1$ and $n \geq 1$ (see [3]). Therefore, the dual sequences (see [16]) are equal in distribution to the PGFs of reproduction by $n$ cycles:

$$
\begin{aligned}
\phi_{n}^{\leftarrow}(s, \sigma) & :=\varphi_{n} \circ \varphi_{n-1} \circ \cdots \circ \varphi_{1}(s, \sigma) \stackrel{\mathrm{D}}{=} \phi_{n}(s, \sigma), \\
\psi_{n}^{\leftarrow}\left(s, \sigma^{\prime}\right) & :=\psi_{n} \circ \psi_{n-1} \circ \cdots \circ \psi_{1}\left(s, \sigma^{\prime}\right) \stackrel{\mathrm{D}}{=} \psi_{n}\left(s, \sigma^{\prime}\right) .
\end{aligned}
$$

(c) In our model, the random environments $\sigma$ and $\sigma^{\prime}$ are uniformly mixing stationary processes; see [3] for a definition.

\subsection{Extinction events}

From (5) and (6), we have the following increasing sequences for the events of extinction by $n$ cycles:

$$
\begin{gathered}
\left\{X_{n}=0\right\} \subset\left\{Y_{n}=0\right\} \subset\left\{X_{n+1}=0\right\} \subset \cdots, \\
\left\{X_{n}^{\prime}=0\right\} \subset\left\{Y_{n+1}^{\prime}=0\right\} \subset\left\{X_{n+1}^{\prime}=0\right\} \subset \cdots .
\end{gathered}
$$

The unconditional probabilities of extinction are defined by the constants

$$
\begin{aligned}
q(X, Y): & =\mathrm{P}\left(X_{n}=0 \text { for some } n=1,2, \ldots\right)=\mathrm{P}\left(Y_{n}=0 \text { for some } n=1,2, \ldots\right), \\
q\left(X^{\prime}, Y^{\prime}\right): & =\mathrm{P}\left(X_{n}^{\prime}=0 \text { for some } n=1,2, \ldots\right)=\mathrm{P}\left(Y_{n}^{\prime}=0 \text { for some } n=1,2, \ldots\right) .
\end{aligned}
$$

The conditional probabilities of extinction, given the environments $\sigma$ and $\sigma^{\prime}$, are defined by the random variables $Q$ and $Q^{\prime}$ as follows:

$$
\begin{aligned}
Q & =\mathrm{P}\left(Y_{n}=0 \text { for some } n=1,2, \ldots \mid \sigma\right)=\lim _{n \rightarrow \infty} \phi_{n}(0, \sigma) \quad \text { (a.s.), } \\
Q^{\prime} & \left.=\mathrm{P}\left(X_{n}^{\prime}=0 \text { for some } n=1,2, \ldots \mid \sigma^{\prime}\right)=\lim _{n \rightarrow \infty} \psi_{n}\left(0, \sigma^{\prime}\right) \quad \text { (a.s. }\right) .
\end{aligned}
$$

For the delayed environment of cycles, we have

$$
\begin{gathered}
\mathrm{P}\left(X_{n}=0 \text { for some } n=1,2, \ldots \mid d^{\prime}\right) \stackrel{\mathrm{D}}{=} \lim _{n \rightarrow \infty} f\left(\delta_{1}, \psi_{n-1}\left(0, \sigma^{\prime}\right)\right)=f\left(\delta_{1}, Q^{\prime}\right), \\
\mathrm{P}\left(Y_{n}^{\prime}=0 \text { for some } n=1,2, \ldots \mid d\right) \stackrel{\mathrm{D}}{=} \lim _{n \rightarrow \infty} g\left(\tau_{1}, \phi_{n-1}(0, \sigma)\right)=g\left(\tau_{1}, Q\right) .
\end{gathered}
$$

By monotonicity, these convergences are almost sure. However, the delayed cycles satisfy

$$
\left(\delta_{i}, \tau_{i+1}\right) \stackrel{\mathrm{D}}{=}\left(\delta_{i}, \tau_{i}\right) \quad \text { and } \quad\left(\tau_{i}, \delta_{i+1}\right) \stackrel{\mathrm{D}}{=}\left(\tau_{i}, \delta_{i}\right), \quad i=1,2, \ldots
$$

Consequently,

$$
Q \stackrel{\mathrm{D}}{=} f\left(\delta, Q^{\prime}\right) \quad \text { and } \quad Q^{\prime} \stackrel{\mathrm{D}}{=} g(\tau, Q) .
$$

Denote by $Q_{0}$ and $Q_{0}^{\prime}$ the random variables representing the minimal solutions of the equations $\varphi(s, \sigma)=s$ and $\psi\left(s, \sigma^{\prime}\right)=s$. These are given by

$$
f\left(\delta, g\left(\tau, Q_{0}\right)\right)=Q_{0} \quad \text { and } \quad g\left(\tau, f\left(\delta, Q_{0}^{\prime}\right)\right)=Q_{0}^{\prime} \quad \text { (a.s.). }
$$

Then $Q_{0}=f\left(\delta, Q_{0}^{\prime}\right)$ and $Q_{0}^{\prime}=g\left(\tau, Q_{0}\right)$, a.s. From (8), we have

$$
Q \stackrel{\mathrm{D}}{=} Q_{0} \quad \text { and } \quad Q^{\prime} \stackrel{\mathrm{D}}{=} Q_{0}^{\prime} .
$$


Example 2. Let $f(t, s)$ and $g(t, s)$ be fractional linear PGFs. Then

$$
Q_{0}=1-\frac{\exp \left\{u^{\prime}(1) \delta+v^{\prime}(1) \tau\right\}-1}{\mathrm{e}^{v^{\prime}(1) \tau}\left[\mathrm{e}^{u^{\prime}(1) \delta}-1\right] u^{\prime \prime}(1) / 2 u^{\prime}(1)+\left[\mathrm{e}^{v^{\prime}(1) \tau}-1\right] v^{\prime \prime}(1) / 2 v^{\prime}(1)} \quad \text { a.s.). }
$$

Clearly,

$$
q(X, Y)=\mathrm{E}(Q) \quad \text { and } \quad q\left(X^{\prime}, Y^{\prime}\right)=\mathrm{E}\left(Q^{\prime}\right) .
$$

Definition 1. (Critical parameters.) We define the constants $m$ and $\gamma$ as follows:

$$
\begin{aligned}
m & :=\mathrm{E}\left(\varphi^{\prime}(1, \sigma)\right)=\mathrm{E}\left(\psi^{\prime}\left(1, \sigma^{\prime}\right)\right)=\mathrm{E}\left(\exp \left\{u^{\prime}(1) \delta+v^{\prime}(1) \tau\right\}\right), \\
\gamma & :=\mathrm{E}\left(\log \varphi^{\prime}(1, \sigma)\right)=\mathrm{E}\left(\log \psi^{\prime}\left(1, \sigma^{\prime}\right)\right)=\mathrm{E}\left(u^{\prime}(1) \delta+v^{\prime}(1) \tau\right) .
\end{aligned}
$$

The reproductions by one cycle $\zeta \stackrel{\mathrm{D}}{=} \zeta_{n}$ and $\zeta^{\prime} \stackrel{\mathrm{D}}{=} \zeta_{n}^{\prime}$ will be labeled supercritical, critical, or subcritical if $\gamma>0, \gamma=0$, or $\gamma<0$, respectively.

Jensen's inequality implies that $m \geq \mathrm{e}^{\gamma}$ for all $m$ and $\gamma$.

Example 3. If the observation and treatment times, being mutually independent, follow the exponential distributions mentioned in Proposition 4, then, for $\alpha>u^{\prime}(1)$,

$$
m=\frac{\alpha \beta}{\left[\alpha-u^{\prime}(1)\right]\left[\beta-v^{\prime}(1)\right]} \quad \text { and } \quad \gamma=\frac{u^{\prime}(1)}{\alpha}+\frac{v^{\prime}(1)}{\beta} .
$$

The classical Galton-Watson processes with reproduction laws given by the unconditional PGFs $\bar{\varphi}, \bar{\psi}, \bar{f} \circ \bar{g}$, and $\bar{g} \circ \bar{f}$ have the constant $m$ as critical parameter, while, in the model of autoregressive control, $m=f^{\prime}(1) \mathrm{E}\left(\exp \left\{v^{\prime}(1) \tau\right\}\right)$ and $\gamma=\log \left[f^{\prime}(1)\right]+v^{\prime}(1) \mathrm{E}(\tau)$. The unconditional means of the BPREs $\left(X_{n}\right)$ and $\left(Y_{n}^{\prime}\right)$ are given by $\mathrm{E}\left(Y_{n}\right)=\mathrm{E}\left(X_{n}^{\prime}\right)=m^{n}$.

Theorem 1. Assume that $u^{\prime}(1)>0$ and $v^{\prime}(1)<0$, and that the random variables $\tau$ and $\delta$ have finite means.

- If $\gamma \leq 0$ then $Q=Q^{\prime}=1$ (a.s.).

- If $\gamma>0$ and if, additionally, $\mathrm{P}(\tau>0)=\mathrm{P}(\delta>0)=1$ and

$$
\mathrm{E}(-\log (1-g(\tau, f(\delta, 0))))<\infty, \quad \mathrm{E}(-\log (1-f(\delta, g(\tau, 0))))<\infty,
$$

then

$$
\begin{gathered}
q<Q<1 \text { and } q<Q^{\prime}<1 \quad(\text { a.s. }), \\
\mathrm{P}\left(Q^{\prime} \leq x\right)<\mathrm{P}(Q \leq x) \text { for all } x, q<x<1,
\end{gathered}
$$

where the constant $q$ is the extinction probability of the supercritical Markov branching process $\xi(t)$.

Proof. The case $\gamma \leq 0$ is well known; see [2, Corollary 1]. Consider the supercritical case. By (9), following [2, Theorem 3], we have

$$
\mathrm{P}\left(Q_{0}<1\right)=\mathrm{P}(Q<1)=\mathrm{P}\left(Q_{0}^{\prime}<1\right)=\mathrm{P}\left(Q^{\prime}<1\right)=1 .
$$

We intend to compare two convex functions having common tangent at the point $s=1$. Let $R(s)=g(T, f(t, s))-f(t, g(T, s))$ for deterministic $T>0$ and $t>0$. Obviously, for each 
$s, q \leq s<1$, we have $R^{\prime}(s)<0$ and $R(s)>0$. The assumption $\mathrm{P}(\tau>0)=\mathrm{P}(\delta>0)=1$ almost surely guarantees the convexity of $f(\delta, s)$ and the truth of the following inequalities:

$$
f(\delta, s)<f(\delta, g(\tau, s))<g(\tau, f(\delta, s))<g(\tau, s) \quad \text { (a.s.) for each } s, q \leq s<1 .
$$

By monotonicity and convexity of the PGF we can find almost-sure bounds on the roots of (8):

$$
f(\delta, g(\tau, q))<Q_{0}, \quad g(\tau, q)<Q_{0}^{\prime} \quad \text { (a.s.). }
$$

Since $q<g(\tau, q)$ (a.s.), we have

$$
\mathrm{P}(q<Q)=\mathrm{P}\left(q<Q_{0}\right)=\mathrm{P}\left(q<Q_{0}^{\prime}\right)=\mathrm{P}\left(q<Q^{\prime}\right)=1 .
$$

Moreover, $f\left(\delta, Q_{0}\right)<Q_{0}<g\left(\tau, Q_{0}\right)$ (a.s.) since $q<Q_{0}<1$ (a.s.). From the definition (8) of the random variables $Q_{0}$ and $Q_{0}^{\prime}$, we have $g\left(\tau, Q_{0}\right)=Q_{0}^{\prime}$ (a.s.). Thus,

$$
q<Q_{0}<Q_{0}^{\prime}<1 \quad \text { (a.s.) }
$$

and, consequently,

$$
\mathrm{P}\left(Q^{\prime} \leq x\right)=\mathrm{P}\left(Q_{0}^{\prime} \leq x\right)<\mathrm{P}\left(Q_{0} \leq x\right)=\mathrm{P}(Q \leq x) \text { for all } x, q<x<1 .
$$

Remark 1. Naturally, setting $m \leq 1$ causes extinction with probability one, i.e. $q(X, Y)=$ $q\left(X^{\prime}, Y^{\prime}\right)=1$. However, the most precise sufficient condition for extinction is given by the critical parameter $\gamma$. If $\mathrm{E}(\delta)=\infty$ and $\mathrm{E}(\tau)<\infty$, then $\mathrm{E}(-\log (1-f(\delta, g(\tau, 0))))<\infty$ implies that $\mathrm{P}(Q=1)=0$. The unresolved cases are $\mathrm{E}(\delta)=\infty$ and $\mathrm{E}(\tau)=\infty$, or $\gamma>0$ and $\mathrm{E}(-\log (1-f(\delta, g(\tau, 0))))=\infty($ see $[4$, p. 255$])$.

\section{Limit theorems}

We now consider the asymptotic behavior of the BPRE $\left(Y_{n}\right)$ generated by the reproduction $\zeta$ by the cycles (observation, treatment). The symmetry with $\left(X_{n}^{\prime}\right)$, describing (treatment, observation), is obvious. Our results are particular cases of those proved in [3]. We outline the specific features of a random environment and PGFs. The main normalizing quantity is the conditional mean of the reproduction by $n$ cycles, i.e.

$$
M_{n}:=\prod_{i=1}^{n} \varphi_{i}^{\prime}(1, \sigma)=\exp \left\{\sum_{i=1}^{n} u^{\prime}(1) \delta_{i}+v^{\prime}(1) \tau_{i}\right\} .
$$

Theorem 2. (Supercritical case.) Assume that $\mathrm{E}(-\log (1-f(\delta, g(\tau, 0))))<\infty$. Let $W_{n}=$ $Y_{n} / M_{n}$, and denote by $\mathbb{F}_{n}(\sigma)$ the filtration generated by the random variables $\left(Y_{1}, Y_{2}, \ldots, Y_{n}\right)$ and the random environment $\sigma$. If $\gamma>0$ then the family

$$
\left\{W_{n}, \mathbb{F}_{n}(\sigma), n=1,2, \ldots\right\}
$$

constitutes a nonnegative martingale and, hence,

$$
\lim _{n \rightarrow \infty} W_{n}=W \quad \text { exists (a.s.). }
$$

Suppose, in addition, that

$$
\mathrm{E}\left(\frac{\mathrm{E}(\zeta \log \zeta \mid \sigma)}{\varphi^{\prime}(1, \sigma)}\right)<\infty
$$


Then the limit

$$
\lim _{n \rightarrow \infty} \mathrm{E}\left(\mathrm{e}^{-\lambda W_{n}} \mid \sigma\right)=: w(\lambda, \sigma), \quad \lambda>0,
$$

exists (a.s.) and is the unique solution of the functional equation

$$
w(\lambda, \sigma)=f\left(\delta_{1}, g\left(\tau_{1}, w\left(\frac{\lambda}{\varphi_{1}^{\prime}(1, \sigma)}, T \sigma\right)\right)\right) \quad \text { (a.s.), }
$$

among those satisfying

$$
\lim _{\lambda \downarrow 0} \frac{1-w(\lambda, \sigma)}{\lambda}=1 \quad \text { (a.s.). }
$$

Moreover, $\mathrm{E}(W \mid \sigma)=1$ and $\mathrm{P}(W=0 \mid \sigma)=Q$ (a.s.).

Theorem 3. (Subcritical case.) Assume that $\mathrm{E}(-\log (1-f(\delta, g(\tau, 0))))<\infty$. If $\gamma<0$ then, for each $s, 0 \leq s<1$,

$$
\lim _{n \rightarrow \infty} \mathrm{E}\left(s^{Y_{n}} \mid Y_{n} \neq 0, \sigma\right) \stackrel{\mathrm{D}}{=} 1-\mathcal{F}(s, \sigma)
$$

Here, the random function $\mathcal{F}(s, \sigma)$ obeys the relation

$$
\mathcal{F}(s, \sigma) \mathcal{F}\left(\varphi_{1}(0, \sigma), T \sigma\right) \stackrel{\mathrm{D}}{=} \mathcal{F}\left(\varphi_{1}(s, \sigma), T \sigma\right) .
$$

The values of $\mathcal{F}(s, \sigma)$ at the points $s=\varphi_{1}(0, \sigma)$ and $s=1$ are as follows:

$$
\mathcal{F}\left(\varphi_{1}(0, \sigma), T \sigma\right)=\varphi_{1}^{\prime}(1, \sigma), \quad \mathcal{F}(1-, \sigma)=0 \quad(\text { a.s. }) .
$$

Theorem 4. (Critical case.) Assume that

$$
\left.0<c_{1} \leq \varphi_{1}^{\prime}(1, \sigma), \quad 0<c_{2} \leq \varphi_{1}^{\prime \prime}(1, \sigma), \quad \varphi_{1}^{\prime \prime \prime}(1, \sigma) \leq c_{3} \quad \text { (a.s. }\right)
$$

and let

$$
a_{n}(\sigma)=\frac{1}{2} \frac{\phi_{n}^{\leftarrow \prime \prime}(1, \sigma)}{\phi_{n}^{\leftarrow \prime}(1, \sigma)}=\frac{1}{2} \sum_{i=1}^{n} M_{i-1} \frac{\varphi_{i}^{\prime \prime}(1, \sigma)}{\varphi_{i}^{\prime}(1, \sigma)}
$$

If $\gamma=0$ then

$$
\left(\frac{Y_{n}}{a_{n}(\sigma)} \mid Y_{n} \neq 0, \sigma\right)
$$

converges in law to an exponentially distributed random variable.

\section{Acknowledgement}

The author is very grateful to an anonymous referee for valuable comments on the paper.

\section{References}

[1] Aly, E.-E. A. AND Bouzar, N. (1994). Explicit stationary distributions for some Galton-Watson processes with immigration. Commun. Statist. Stoch. Models 10, 499-517.

[2] Athreya, K. B. And Karlin, S. (1971). On branching processes with random environments. I. Extinction probabilities. Ann. Math. Statist. 42, 1499-1520.

[3] Athreya, K. B. and Karlin, S. (1971). Branching processes with random environments. II. Limit theorems. Ann. Math. Statist. 42, 1843-1858.

[4] Athreya, K. B. And Ney, P. E. (1972). Branching Processes. Springer, Berlin. 
[5] Borovkov, A. A. et al. (2004). On the 80th birthday of B. A. Sevastyanov. Theory Prob. Appl. 48, 697-702.

[6] Del Puerto, I. M. And Yanev, N. M. (2004). Branching processes with multi-type random control functions. C. R. Acad. Bulgare Sci. 57, 29-36.

[7] GonzÀlez, M., Molina, M. And Del Puerto, I. (2002). On the class of controlled branching processes with random control functions. J. Appl. Prob. 39, 804-815.

[8] Gonzàlez, M., Molina, M. And Del Puerto, I. (2003). On the geometric growth in controlled branching processes with random control functions. J. Appl. Prob. 40, 995-1006.

[9] Gonzàlez, M., Molina, M. And Del Puerto, I. (2004). Limiting distribution for subcritical controlled branching processes with random control functions. Statist. Prob. Lett. 67, 277-284.

[10] Gonzàlez, M., Molina, M. and Del Puerto, I. (2005). On $L_{2}$-convergence of controlled branching processes with random control functions. Bernoulli 11, 37-46.

[11] Kuimov, G. P. (1983). Theory of Probability and Mathematical Statistics. Moscow State University Press (in Russian).

[12] McDonald, D. (1978). On semi-Markov and semi-regenerative processes. II. Ann. Prob. 6, 995-1015.

[13] PAKes, A. G. (1995). Characterisation of discrete laws via mixed sums and Markov branching processes. Stoch. Process. Appl. 55, 285-300.

[14] Sevast'yanov, B. A. (1971). Branching Processes. Nauka, Moscow.

[15] Sevast'yanov, B. A. And Zubkov, A. M. (1974). Controlled branching processes. Theory Prob. Appl. 19, 14-24.

[16] Smith, W. L. AND Wilkinson, W. E. (1969). On branching processes in random environments. Ann. Math. Statist. 40, 814-827.

[17] Steutel, F. W. and van Harn, K. (1979). Discrete analogues of self-decomposability and stability. Ann. Prob. 7, 893-899.

[18] Van Harn, K. and Steutel, F. W. (1993). Stability equations for processes with stationary independent increments using branching processes and Poisson mixtures. Stoch. Process. Appl. 45, 209-230.

[19] Yanev, N. M. (1976). Conditions for degeneracy of $\varphi$-branching processes with random $\varphi$. Theory Prob. Appl. 20, 421-428. 